\title{
PROCESS AND SYSTEMS How to improve medication safety at hospital discharge: let's get practical
}

\author{
Author: Jennifer Flatman ${ }^{A}$
}

\begin{abstract}
The hospital discharge transition is a well-known risk factor for medication-related harm. The Royal College of Physicians have created an improvement guide and resource, focusing on medication safety at hospital discharge. In this article, we discuss how we developed this resource and explore some of the lessons learnt, including the importance of involving patients and carers; understanding the discharge medicines system; appreciating everyone's roles; measuring medication safety, project spread and sustainability; and the pros and cons of a virtual working environment.
\end{abstract}

KEYWORDS: patient safety, medication safety, hospital discharge, transitions of care, quality improvement

DOI: 10.7861/fhj.2021-0176

\section{Introduction}

Medicines benefit many patients, yet their use can also carry considerable risk. It is estimated that $50 \%$ of adult patients are affected by medication errors and almost $20 \%$ experience an adverse drug event post-hospital discharge. ${ }^{1}$ Not all medication errors lead to patient harm but even those that don't, can still reduce efficacy and patients' confidence in the healthcare system. ${ }^{2}$

Reducing medication-related harm is an international priority and transitions of care has been identified as a key action area. ${ }^{3}$ Hospital discharge is one such transition and can be defined as the various points where a patient moves to or returns from a particular physical location or makes contact with a health care professional for the purposes of receiving health care. ${ }^{3}$ Effective transitions from hospital to community are as important as the care a patient receives as an inpatient and many organisations have recognised the importance of targeting medicines use at transitions of care to improve medication safety. ${ }^{3-6}$ Even though improving medication safety when individuals are discharged from hospital has been a subject of interest for at least a decade, recent publications show we still face issues and potential harms from medication at this common care transition. $1,5,7,8$

As part of the global patient safety challenge, Medication Without Harm, the World Health Organization (WHO) identified five

Author: Amedicines safety clinical fellow, Royal College of Physicians, London, UK key priorities for improving medication safety at care transitions, which we adapted for the hospital discharge transition. ${ }^{3}$

> Implementing formal structured processes for medicines reconciliation when patients are being discharged from hospital.

> Partnering between patients, caregivers and healthcare professionals at hospital discharge.

> Prioritising patients at a high risk of medication-related harm around hospital discharge.

> Implementing collaborative medicines optimisation at hospital discharge.

> Improving the quality and availability of medication-related information at hospital discharge.

Each of these is a key target for improving medication safety at hospital discharge and a useful starting point for developing any improvement project in this area.

Medicine classes most commonly reported with adverse drug events post-hospital discharge are cardiovascular, analgesic, antibiotic and antidiabetic medicines, and so could be worthwhile targets for improvement projects. 'Research also shows that the rate of medication errors and adverse drug events at the hospital discharge transition is higher for older people. Combining interventions to improve medication safety at hospital discharge for older people is more effective than compared with single interventions and interventions that bridge care transitions can support medication continuity and help to reduce hospital readmissions.

There is still much progress to be made in improving the safety of medicines use for patients, carers, families and healthcare staff at the hospital discharge transition, but what can we do about it?

\section{Developing the Royal College of Physicians'} medication safety at hospital discharge improvement guide and resource

We set out to use our expertise in quality improvement (QI), medication safety, patient-centred multidisciplinary working and ward processes to help healthcare organisations answer this question by bringing together tools and information to guide a team through an improvement project focusing on medication safety at hospital discharge. Our aims were:

$>$ to raise awareness of the potential harms caused by medication errors when patients are discharged from hospital

$>$ to encourage secondary care organisations to create and implement their own improvement projects to optimise local discharge processes for reducing medication-related harm > to improve interprofessional collaboration and learning. 
We have created an improvement guide and resource (www.rcplondon.ac.uk/projects/outputs/medication-safetyhospital-discharge-improvement-guide-and-resource) based on QI methods to help teams make valuable and sustainable improvements to medication safety when people are discharged.

When developing the guide, promoting interprofessional collaboration was a key priority, as everyone needs to play a role in improving medication safety. The guide is aimed at project teams based in a hospital environment but emphasises the need to include staff and patients from other health and social care sectors, including primary care. It was also important that we didn't look at medications in silo but as part of the whole discharge system to reflect clinical practice.

We started by conducting a literature search of QI work which focused on improving medication safety at the hospital discharge transition. Many were small, local case studies but this meant they were more likely to be able to be replicated by our main audience: small project teams within a hospital. As many healthcare organisations use the Model for Improvement for QI projects, we used this as a basis for our guide. ${ }^{10}$ This model is based on three questions.

$>$ What are we trying to accomplish?

> How will we know that a change is an improvement?

> What change can we make that will result in improvement?

This model also uses plan, do, study, act (PDSA) cycles to test and adapt interventions on a small scale before introducing them more widely. Even though we used the Model for Improvement, we made sure that the guide can be used with any improvement model the team chooses. The guide follows a logical structure (Fig 1) and key elements of the guide include:

$>$ QI tools and templates

$>$ practical examples relating to this topic eg a process map of an example discharge medicines pathway, a cause and effect diagram exploring why warfarin may be omitted from discharge medication lists
> bitesize exercises and extra optional activities should the team need to spend more time on a particular area of the QI project.

We tested the guide in a pilot phase, with volunteer teams from NHS trusts in different parts of the UK. Many teams used the guide to start implementing QI projects within their organisations and gave feedback on their experience and the guide.

\section{Learning from developing the guide}

By developing this guide, we've learnt valuable lessons, many of which will also apply to other improvement projects.

Patient safety, the clue is in the name: the importance of involving patients and carers at every step

Patients, carers and families are integral to improving medication safety at care transitions. We worked with the Royal College of Physician's Patient and Carer Network and health and social care stakeholders from a wide range of sectors and backgrounds. We strongly recommend that core project teams include patients or patient representatives; measures include patient and carer perspectives; and interventions are designed with patients and carers. Ensuring patients are front and centre in patient safety improvements is not a new concept and improving patient involvement in their own safety is a national priority. ${ }^{11}$ Patients may require more support to be involved in their own medication safety at hospital discharge and this improvement guide can help. ${ }^{12}$

\section{Understanding the system}

It's hard to know where to make improvements without appreciating how the system you're looking to make improvements in currently works. One of the first tools introduced in the guide is called process mapping. Many are familiar with this type of tool, also called flow charts, but may not have thought to use it to explore what happens when people are discharged

Section 1: Introduction

Looks at creating a project team, arranging project meetings and gives advice on deciding a QI approach

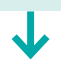

Section 2: Identifying the problems

Helps the team to gain an understanding of the local discharge medicines system, potential issues to focus on and who should be involved in the project

Section 3: Measuring medication safety at hospital discharge

Explains why measuring for improvement is important and includes potential ways to measure medication safety at hospital discharge

Section 4: Defining the project

Explores ways to define the scope of the project, including the area of focus and improvement interventions

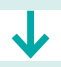

Section 5: Implementing your improvements

Introduces PDSA cycles and how to sustain and spread the project in the long term

Fig 1. Improvement guide and resource overview. PDSA = plan, do, study, act; QI = quality improvement. 
with medicines. Given that the discharge medicines pathway can be long and complex, this is an important tool to help the team understand the local discharge medicines pathway. Involving multiple people with different views and experiences is important for obtaining a useful and realistic process map. Everyone's views are important, one person won't know what happens at every part of the pathway but together we can begin to understand the complete picture.

\section{Appreciating everyone's roles}

The guide was created by a multidisciplinary team and was made to help teams encourage interprofessional collaboration within and outside of their organisations, including patients and carers and their representatives. We can make a lot of assumptions about what those involved in the discharge medicines pathway can and can't do but without understanding and appreciating the roles of everyone involved, we won't fully understand the system and, therefore, may miss useful improvement interventions. We included a roles and responsibilities matrix for this reason, to encourage team members to understand who does what and when.

\section{How do we measure medication safety?}

Measuring medication safety can be a challenge but measuring for improvement is an important element of any QI project and we include key points and examples of ways to measure medication safety in the guide. Using both qualitative and quantitative data helps to give a more holistic approach to measurement and including patient oriented measures (such as patient experience or outcome measures that reflect the impact on the patient) is especially important to find out whether our improvements are benefiting patients. It requires continuous data to show improvement, so automating data collection is important to ensure sustainability, rather than having to manually collect and analyse data.

\section{Spread and sustainability}

Not to be underestimated, planning spread and sustainability into improvement projects is as important as starting. Once an intervention is found to work, you need to know how you will ensure these interventions can be spread on a larger scale. Successive PDSA cycles can help to gradually spread your intervention and adapt where needed. Once you have introduced your interventions on a larger scale, you also need to plan how the interventions will be sustained by embedding the actions and measurement into usual practice.

\section{Virtual working environments: pros and cons}

Many of us will have experienced at least one virtual meeting over the past 2 years and so can understand the benefits and challenges. Virtual meetings may be easier to arrange, as group members can be in any location and time is not taken up by travelling, and sharing screens and documents as presentation aids can really bring an extra dimension to the discussion. Challenges include establishing relationships among a newly formed team over a virtual platform. Opportunities for small talk and elements of body language may be lost, and so team building exercises need to be designed into the meeting, such as ice breakers or breakout sessions. Virtual meetings also require technology and the skills to use them, leading to a risk of excluding those who do not possess such resources.

\section{Conclusion}

While it is important to have a plan in place, there are many competing priorities within healthcare and there will never be a perfect time to start an improvement project. The improvement guide and resource makes it as easy as possible. Improving medication safety at the hospital discharge transition is an important and effective way of improving patient safety, as well as improving links between local health and social care sectors. Quality improvement is a key element of healthcare, and learning how to use QI tools and techniques to make improvements in medication safety at hospital discharge will enhance knowledge that can be used in future projects.

\section{Funding}

Financial support for the medicines safety clinical fellow was provided as an educational grant by Pfizer.

\section{References}

1 Alqenae FA, Steinke D, Keers RN. Prevalence and nature of medication errors and medication-related harm following discharge from hospital to community settings: a systematic review. Drug Saf 2020:43:517-37.

2 Franklin BD and Tully MP. Safety in medication use. CRC Press, 2016.

3 World Health Organization. Medication safety in transitions of care: Technical document. WHO, 2019. https://apps.who.int/iris/ bitstream/handle/10665/325453/WHO-UHC-SDS-2019.9-eng. pdf? sequence $=1$ \&isAllowed =y [Accessed 28 September 2021] .

4 American Society of Health-System Pharmacists, American Pharmacists Association. ASHP-APhA medication management in care transitions best practices. ASHP, 2013.

5 Picton C, Wright H. Keeping patients safe when they transfer between care providers - getting the medicines right. London: Royal Pharmaceutical Society, 2012.

6 Care Quality Commission. Medicines in health and adult social care. Care Quality Commission, 2019.

7 Care Quality Commission. Inpatient experience during the COVID-19 pandemic. Care Quality Commission, 2020.

8 Healthwatch England, British Red Cross. 590 people's stories of leaving hospital during COVID-19. Healthwatch England, 2020.

9 Tomlinson J, Cheong V-Lin, Fylan B et al. Successful care transitions for older people: a systematic review and meta-analysis of the effects of interventions that support medication continuity. Age Ageing 2020;49:558-69.

10 Institute for Healthcare Improvement. How to improve. IHI, 2021. www.hi.org/resources/Pages/HowtoImprove/default.aspx [Accessed 15 October 2021].

11 NHS England, NHS Improvement. Framework for involving patients in patient safety. NHS, 2021. www.england.nhs.uk/wp-content/ uploads/2021/06/B0435-framework-for-involving-patients-inpatient-safety.pdf [Accessed 27 September 2021].

12 Grimes TC. Is it time for greater patient involvement to enhance transitional medication safety? BMJ Qual Saf 2021;bmjqs-2021014116 [Epub online ahead of print].

13 NHS Institute for Innovation and Improvement. The how-to guide for measurement for improvement. NHS, 2008.

Address for correspondence: Jennifer Flatman, Royal College of Physicians, 11 St Andrews Place, Regent's Park, London NW1 4LE, UK.

Email: jennifer.flatman@rcp.ac.uk 